\title{
Mini-symposium
}

\section{Aortic intramural haematoma: introduction}

A ortic intramural haematoma (IMH) has been considered a precursor of overt aortic dissection; however, the pathophysiologic mechanism, evolution, and prognosis are rather different from classical dissection. Advances in imaging techniques have facilitated diagnosis and have aided understanding of the natural history of IMH. The considerable information obtained recently on this entity, practically unknown 10 years ago, warrants this mini-symposium.

Jae-Kwan Song, from Seoul, South Korea, comments on the most important diagnostic findings with the use of different imaging techniques. A notable aspect of his discussion is the differential diagnosis with other aortic processes, and the role of these techniques in the follow up. Christoph Nienaber, from Rostock, Germany, describes the natural evolution of IMH and the predictive factors of progression and mortality. Finally, Michael Dake, from Stanford, California, explains his point of view on the therapeutic management of this entity.

At the end of the three chapters, I comment on the most salient aspects of each presentation and contrast them with the experience of our group. I hope that the outstanding knowledge of the contributors to this mini-symposium will greatly aid in improving the diagnosis and management of this entity, which has clearly differential aspects compared other acute aortic syndromes.

A Evangelista Servei de Cardiologia, Hospital Vall d'Hebron $P^{\circ}$ Vall D'Hebron, Barcelona, Spain; evangel@hg.vhebron.es

\section{IMAGES IN CARDIOLOGY}

\section{Double right aortic sinus inlet of the right coronary artery}

A 56 year old woman with atypical chest pain and both ECG and echocardiographic evidence of lateral ischaemia underwent cardiac catheterisation in our laboratory. Left coronary artery angiography showed critical stenosis of the proximal circumflex artery. Subsequently, using a right Judkins catheter, a right coronary artery ostium was engaged (panel A). This ostium was located low in the right coronary sinus. Interestingly, upon

injection of contrast, the dye returned in the aorta via a (higher) true right coronary ostium (panel B). At first, the operator suspected perforation of the aortic sinus into the right coronary artery. However, additional injections and digital angiography (panel C) confirmed the presence of a double inlet into the right coronary artery. To our knowledge, this is the first description of this rare and benign anatomic anomaly.

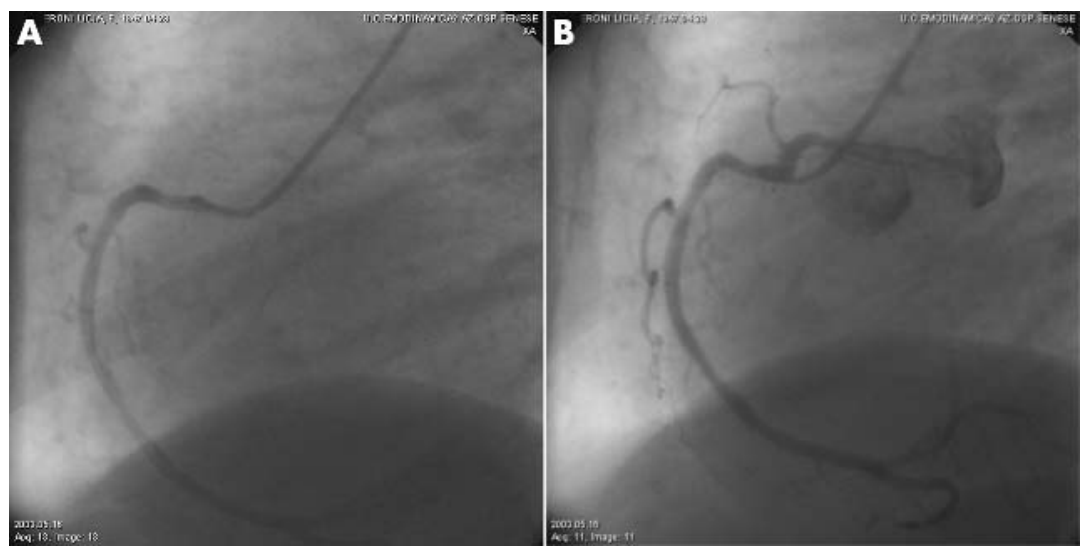

Figure 1 A right Judkins catheter engaged in the lower right coronary artery ostium (A). The dye is injected into the right coronary artery and backflows into the aortic sinus via the upper ostium (B).
The lesion in the circumflex coronary artery was treated with angioplasty and stenting. The patient was discharged from hospital and is currently asymptomatic.
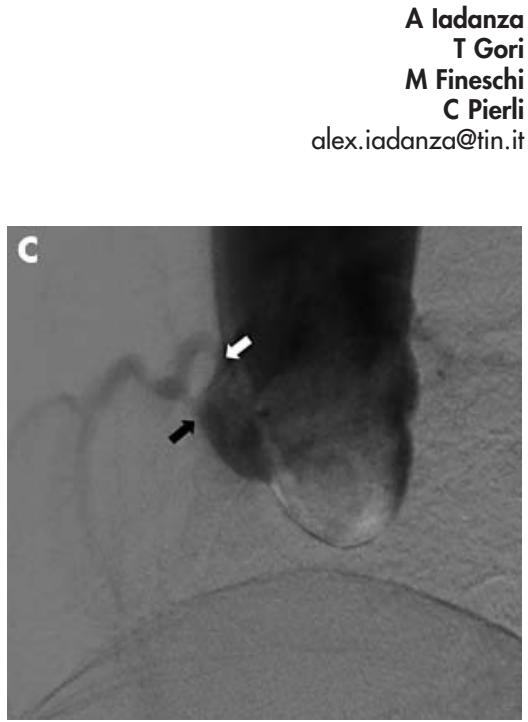

Figure 2 Digital angiography showing the double ostia of the right coronary artery. White arrow shows true right coronary ostium; black arrow shows anomalous, low, right coronary ostium. 